\title{
Relationship of visit-to-visit and ambulatory blood pressure variability to vascular function in African Americans
}

\begin{abstract}
Keith M Diaz ${ }^{1}$, Praveen Veerabhadrappa ${ }^{1}$, Mohammed A Kashem ${ }^{2}$, Deborah L Feairheller ${ }^{3}$, Kathleen M Sturgeon ${ }^{1}$, Sheara T Williamson ${ }^{1}$, Deborah L Crabbe ${ }^{2}$ and Michael D Brown ${ }^{1,4}$

Visit-to-visit clinic blood pressure variability (BPV) and 24-h BPV have both been identified as independent risk factors for cardiovascular (CV) morbidity and mortality; however, the mechanisms contributing to the increased CV risk as yet are unclear. The purpose of this study was to assess the relationship between BPV and endothelial function in a cohort of putatively healthy African Americans. A total of 36 African Americans who were sedentary, non-diabetic, non-smoking, free of CV and renal disease and not on antihypertensive medication followed an American Heart Association low fat, low salt diet for 6 weeks. Upon completion of the 6-week dietary stabilization period, participants underwent 24-h ambulatory BP monitoring and had their office blood pressure (BP) measured on 3 separate days. Right brachial artery diameter was assessed at rest, during reactive hyperemia (flow-mediated vasodilation: FMD), and after nitroglycerin administration (nitroglycerin-mediated vasodilation: NMD). Participants classified as having decreased endothelial function according to either \%FMD or the FMD/NMD ratio had significantly higher 24-h BPV and a trend for higher visit-to-visit BPV when compared with participants with normal endothelial function. Continuous variable analyses revealed a significant positive association between NMD and 24-h diastolic BPV (DBPV). Visit-to-visit systolic BPV (SBPV), 24-h SBPV and 24-h DBPV were all negatively associated with the FMD/NMD ratio. All relationships remained significant after adjustment for age, body mass index and mean BP levels. These results may suggest that BPV is increased in African Americans with decreased endothelial function and is associated with the vascular smooth muscle response to nitric oxide.
\end{abstract}

Hypertension Research (2012) 35, 55-61; doi:10.1038/hr.2011.135; published online 4 August 2011

Keywords: African Americans; ambulatory blood pressure monitoring; blood pressure variability; endothelial function

\section{INTRODUCTION}

For many years, transient fluctuations in blood pressure (BP) were generally thought to not have prognostic importance or mandate treatment in the same way as elevations in mean BP. However, in recent years evidence has accumulated to show that blood pressure variability (BPV) has a striking relationship with cardiovascular (CV) risk and is predictive of future $\mathrm{CV}$ events, independent of mean $\mathrm{BP}$ levels. Two recent examples were provided by data from the Pressioni Arteriose Monitorate E Loro Associazioni (PAMELA) and the Ohasama studies, which both reported that measures of BPV within a 24-h period were independent predictors of CV mortality in the general population. ${ }^{1,2}$ In addition to fluctuations in BP from readingto-reading over a 24 -h period using ambulatory blood pressure monitoring (ABPM), several publications in 2010 and 2011 have shown that visit-to-visit variability in clinic BP is also a powerful predictor of $\mathrm{CV}$ events and all-cause mortality. ${ }^{3,4}$ Most recently, Muntner et al. ${ }^{3}$ via retrospective analysis of the general populationbased Third National Health and Nutrition Examination Survey (NHANES III), showed that visit-to-visit BPV was associated with increased all-cause mortality independent of mean BP levels, as subjects in the middle and highest tertiles of visit-to-visit BPV had a $>50 \%$ greater risk of all-cause mortality than subjects in the lowest tertile of visit-to-visit BPV.

Despite the mounting evidence and growing interest in visit-to-visit and ambulatory BPV, scant attention has been paid to the mechanisms by which high BPV may confer a greater CV risk. It has been suggested that the augmented mechanical stress placed on the vasculature as a result of increased variability of blood flow may induce a subclinical inflammatory vascular response which, in turn, could lead to the impairment of endothelial function. ${ }^{5}$ In support of this hypothesis,

${ }^{1}$ Hypertension, Molecular and Applied Physiology Laboratory, Department of Kinesiology, College of Health Professions, Temple University, Philadelphia, PA, USA; ${ }^{2}$ Division of Cardiology, Department of Medicine, School of Medicine, Temple University, Philadelphia, PA, USA; ${ }^{3}$ Exercise and Metabolic Disease Research Laboratory, Department of Physiological Sciences, University of California, Los Angeles, CA, USA and ${ }^{4}$ Cardiovascular Research Center, School of Medicine, Temple University, Philadelphia, PA, USA Correspondence: KM Diaz, Hypertension, Molecular and Applied Physiology Laboratory, Department of Kinesiology, Temple University, 1800 N. Broad Street, Philadelphia, PA 19122, USA.

E-mail: keith.diaz@temple.edu

Received 15 April 2011; revised 14 June 2011; accepted 17 June 2011; published online 4 August 2011 
we and several others have shown that high $24-\mathrm{h} \mathrm{BPV} \mathrm{is} \mathrm{strongly}$ associated with biomarkers of systemic and vascular inflammation, independent of mean BP levels. ${ }^{5-8}$ However, to the best of our knowledge, no previous study has assessed the relationship between BPV and endothelial function. When considering that impaired endothelial function has been strongly associated with CV morbidity and mortality, ${ }^{9-11}$ the previously observed associations between high BPV and increased CV risk could be mediated via the impairment of endothelial function. The purpose of this study, therefore, was to investigate the association between endothelial function and both visit-to-visit and ambulatory BPV in a cohort of putatively healthy African Americans.

\section{METHODS}

\section{Participants}

Participants were recruited via mailed brochures and local newspaper advertisements. On response to either, participants were contacted by telephone to assess eligibility. Each participant gave written informed consent following explanation of study protocols during their first laboratory visit. The protocol was approved by the Temple University Institutional Review Board.

The study included African Americans between the ages of 40-75 who were sedentary (regular aerobic exercise $<2$ days per week), non-smoking, nonmorbidly obese (body mass index (BMI) $<40 \mathrm{~kg} \mathrm{~m}^{-2}$ ), not on lipid-lowering medication, had a clinic BP $<160 / 100 \mathrm{~mm} \mathrm{Hg}$, and had no history of CV disease, diabetes, hypercholesterolemia, renal disease or lung disease. Both pre$(n=15)$ and postmenopausal $(n=12)$ women were included in the study; all postmenopausal women were not on hormone replacement therapy. Participants on more than one antihypertensive medication were excluded from the study.

\section{Screening}

To ensure the eligibility of all qualified participants, three screening visits were completed before inclusion in the study. Screening visit one consisted of blood sampling and urinalysis following a 12-h overnight fast to assess blood chemistries and renal function. Any individual with total cholesterol $>240 \mathrm{mg} \mathrm{dl}^{-1}$ or fasting blood glucose $>126 \mathrm{mg} \mathrm{dl}^{-1}$ were excluded from the study. Estimated glomerular filtration rate (eGFR) was calculated using the four-variable modification of diet in renal disease (MDRD) study equation specific to African Americans. ${ }^{12}$ Any individual who exhibited evidence of renal disease (eGFR $<60 \mathrm{ml} \mathrm{min}^{-1}$ per $1.73 \mathrm{~m}^{2}$ ) was excluded from the study.

Screening visits two and three required all qualified participants to undergo a physician-administered physical examination and echocardiogram bicycle stress test to confirm that participants displayed no evidence of $\mathrm{CV}$, pulmonary or other chronic diseases.

\section{Dietary stabilization}

In order to rule out the confounding effect of variations in dietary intake, participants who met all inclusion criteria after screening underwent dietary stabilization for 6 weeks before testing. Any participant receiving antihypertensive monotherapy $(n=12)$ was tapered off of their medication during this dietary stabilization period. Participants were instructed by a Registered Dietician on the American Heart Association Dietary Guidelines for Healthy American Adults. ${ }^{13}$ This diet consisted of $\sim 55 \%$ of total daily calories from carbohydrates, $15 \%$ from protein, and $<30 \%$ from fat, with saturated fat $\leqslant 10 \%$ of total calories, sodium $\leqslant 3-4 \mathrm{~g}$ per day, and cholesterol intake $<300 \mathrm{mg}$ per day. Participants met with the dietician once per week at which time body weight was recorded for each visit. Participants were required to remain within $5 \%$ of their study entry body weight for the duration of the study. Compliance to the prescribed diet was monitored by completion of a 3-day food record at the conclusion of dietary stabilization. All participants who were in compliance with the diet underwent testing 1-2 weeks after dietary stabilization.

\section{Office BP measurements}

Office BP measurements were made in accordance with JNC 7 guidelines ${ }^{14}$ on three separate visits by trained laboratory personnel. BP was measured using a mercury sphygmomanometer after $5 \mathrm{~min}$ of quite rest in a chair with feet on the floor and arm supported at heart level. The appropriate size cuff was determined by upper arm circumference. BP measurements were performed in triplicate, $5 \mathrm{~min}$ apart and the average of the three values was used as the BP for the visit. The mean duration between visits 1 and 2 was $7 \pm 1$ days. The mean duration between visits 2 and 3 was $8 \pm 1$ days. Using the mean systolic BP (SBP) and diastolic BP (DBP) from each visit, the s.d. and coefficient of variation for SBP and DBP across study visits were calculated as measures of BPV.

\section{4-H АBPM}

Participants underwent 24-h ABPM using a non-invasive monitor (SpaceLabs Medical, Model 90219, Redmond, WA, USA) beginning on the morning of each participant's typical day, with the exclusion of Friday through Sunday. The BP cuff was fitted to participant's non-dominant arm with cuff size determined by upper arm circumference. BP measurements were obtained at 30-min intervals during the day (0600-2200 hours) and 60-min intervals at night (2200-0600 hours). Participants were instructed not to exercise before or during the monitoring period and to pause momentarily and maintain their body position during each BP measurement. Throughout the duration of the recording period, participants were required to maintain a diary in which they recorded their activity at the time of each BP measurement. Only recordings of good technical quality ( $>80 \%$ of valid BP measurements) were included in final analyses.

\section{Analysis of ABPM data}

Mean values were calculated for 24-h SBP and DBP. BPV was calculated using two different parameters: the average real variability index (ARV) and the time rate of variation index. The ARV index and the time rate of variation index were calculated as previously described. ${ }^{15}$ The rationale for selecting the ARV index for BPV calculations is based on previous studies that have reported the ARV index to be a more reliable representation of time series variability than s.d. or coefficient of variation. ${ }^{16,17}$ We selected the time rate of variation as an additional measure of BPV because it accounts for the order in which the BP measurements are obtained, as well as the time between successive readings. ${ }^{18}$ Moreover, this parameter permits the evaluation of how fast or slow and in which direction $\mathrm{BP}$ values change. The rationale for using both the ARV index and the time rate of variation was to allow us to quantify the absolute magnitude of BPV (ARV index), while also considering the magnitude of BPV in relation to the time that elapsed between successive BP readings (time rate of $\mathrm{BPV}$ ).

\section{Bioelectrical impedance analysis}

Body composition was assessed by whole-body bioelectrical impedance analysis using the single frequency impedance instrument ImpediMed DF50 (San Diego, CA, USA). Bioelectrical impedance analysis was measured at $50 \mathrm{kHz}$ on the right side of the body, with two electrodes placed on each dorsal right hand and dorsal right foot while participants were lying in a supine position with their legs slightly apart and hands resting next to the body palms down. Participants were asked to remove all jewelry and other accessories before measurements. All electrode sites were cleaned with an alcohol swab before attachment. Measures were taken after at least $10 \mathrm{~min}$ of lying in the supine position to reduce possible errors from acute changes in body fluid distribution. Three measurements were taken; and the mean output values of impedance, phase, resistance and reactance were used for calculations of total fat mass and total lean body mass according to the manufacturer's standard operating procedures.

\section{Brachial artery ultrasound assessment of endothelial-dependent and -independent function}

Brachial artery (BA) diameter was measured in response to increased flow (flow-mediated vasodilation: FMD) and in response to nitroglycerin (nitroglycerin-mediated vasodilation: NMD) as previously described. ${ }^{19}$ All measurements were performed in the morning following an overnight fast during which time participants refrained from food, drink (with the exception of water), caffeine, alcohol, antihistamine and anti-inflammatory medications. 
A 7.5-MHz linear phased array ultrasound transducer attached to a Sonos 5500 ultrasound machine (Philips Medical Systems, Bothell, WA, USA) was used to image the BA longitudinally. Electrocardiogram was continuously monitored. All measurements of BA diameter were taken after at least 10 min of lying in the supine position by a trained cardiologist in a quiet and dim room at controlled ambient temperatures $\left(20-26^{\circ} \mathrm{C}\right)$. The participant's right arm was comfortably immobilized in the extending position, allowing for ultrasound scanning of the $\mathrm{BA}$ artery $5-10 \mathrm{~cm}$ above the antecubital fossa. Baseline images of the right BA were first obtained. After recording of baseline images, reactive hyperemia was induced by distal occlusion of the vessel using a cuff inflated to suprasystolic pressure $(200 \mathrm{~mm} \mathrm{Hg})$ for $5 \mathrm{~min}$ on the right forearm, distal to the antecubital fossa as described by Corretti et al. ${ }^{20}$ BA diameter was imaged at $60 \mathrm{~s}$ and recorded for $5 \mathrm{~min}$ post cuff-release. After at least $15 \mathrm{~min}$ of rest, new baseline images were obtained and a $0.4 \mathrm{mg}$ nitroglycerin tablet was given sublingually to assess endothelium-independent vasodilation. Images were then recorded 4 min later.

Offline image analysis was performed by trained personnel blinded to image sequence and participants' clinical data. Arterial diameter was measured from the anterior to the posterior ' $\mathrm{m}$ ' line (the interface between media and adventitia) at end-diastole, incident with the R-wave on the electrocardiogram. $\mathrm{BA}$ vasodilator response to reactive hyperemia and nitroglycerin was calculated as the maximal \% change in BA diameter (FMD: at $60 \mathrm{~s}$; NMD: at $4 \mathrm{~min}$ ) from baseline. As an additional index of endothelial function, the ratio of response to intrinsic nitric oxide (NO)/response to exogenous NO (FMD/NMD ratio) was calculated in order to correct for vascular smooth muscle (VSM) function in each participant.

\section{Statistical analysis}

Data are expressed as means \pm s.e.m. The distribution of all variables was examined using the Shapiro-Wilk test of normality. Variables that were not normally distributed (total cholesterol, high-density lipoprotein cholesterol and triglycerides) were log transformed for statistical analyses, but true physiological values are reported throughout the paper for ease of interpretation. Participants were classified into groups according to their \%FMD, \%NMD and FMD/NMD Ratio. Any participant at or above the median value for the vascular function measure (\%FMD, \%NMD or FMD/NMD ratio) was classified as having normal function; while any participant below the median for the vascular function measure was classified as having decreased function. Comparisons between groups were tested using the independent $t$-test for continuous variables and Pearson's $\chi^{2}$-test for dichotomous variables.

The relationship of each clinical variable and $\mathrm{BP}$ parameter with \%FMD, $\% \mathrm{NMD}$ and the FMD/NMD ratio as continuous variables was tested using univariate regression analyses. All variables significantly associated with $\% \mathrm{FMD}, \% \mathrm{NMD}$ or the FMD/NMD ratio in univariate regression analyses were then inserted separately into a multivariate regression model containing variables known to impact vascular function: age, BMI, 24-h SBP and 24-h DBP. For all BPV indices entered into the model, the corresponding mean BP value was entered into each model (for example, if the dependent variable for a given model was a 24-h BPV variable, the model was adjusted for 24-h SBP and DBP). Each model was evaluated for multicollinearity among variables. The variance inflation factor was $<5$ for all models (range: $1.031-3.247) . P$-values $<0.05$ were considered statistically significant for all analyses. Statistical analyses were performed using SPSS version 17.0 (SPSS, Chicago, IL, USA).

\section{RESULTS}

A total of 36 African Americans who met the study criteria formed the study population. The average age was $52.0 \pm 1.0$ years and $25 \%$ were male. Mean BMI for the entire study group was $32.3 \pm 0.9 \mathrm{~kg} \mathrm{~m}^{-2}$ and the mean total cholesterol was $4.9 \pm 0.1 \mathrm{mmoll}^{-1}$. The mean office SBP and DBP were $125.5 \pm 1.9 \mathrm{~mm} \mathrm{Hg}$ and $79.9 \pm 1.0 \mathrm{~mm} \mathrm{Hg}$, respectively. The mean 24-h SBP and DBP from ABPM were $127.8 \pm 1.8 \mathrm{~mm} \mathrm{Hg}$ and $78.7 \pm 1.4 \mathrm{~mm} \mathrm{Hg}$, respectively. Mean \%FMD for the entire study group was $7.2 \pm 0.4 \%$ and the mean for $\% \mathrm{NMD}$ was $16.1 \pm 0.9 \%$.

Table 1 shows the comparisons between normal and decreased endothelial function groups for all clinical characteristics and BP
Table 1 Clinical characteristics and BP parameters for endothelial function groups classified according to \%FMD

\begin{tabular}{|c|c|c|c|}
\hline Variable & $\begin{array}{c}\text { Normal } \\
\text { endothelial } \\
\text { function } \\
\% F M D \geqslant 7 \% \\
(n=18)\end{array}$ & $\begin{array}{c}\text { Decreased } \\
\text { endothelial } \\
\text { function } \\
\% F M D<7 \% \\
\quad(n=18)\end{array}$ & $\mathrm{P}$-value \\
\hline Age (years) & $51.3 \pm 1.6$ & $52.2 \pm 1.2$ & 0.68 \\
\hline Male $(\%) /$ female (\%) & $\begin{array}{l}7(39 \%) / \\
11(61 \%)\end{array}$ & $\begin{array}{l}2(11 \%) / \\
16(89 \%)\end{array}$ & 0.05 \\
\hline Antihypertensive medication (\%) & 27.7 & 38.8 & 0.48 \\
\hline $\mathrm{BMI}\left(\mathrm{kg} \mathrm{m}^{-2}\right)$ & $31.0 \pm 1.5$ & $34.2 \pm 1.0$ & 0.09 \\
\hline Fat-free mass (kg) & $52.8 \pm 2.5$ & $54.6 \pm 1.8$ & 0.56 \\
\hline Fat mass (kg) & $33.7 \pm 3.2$ & $44.5 \pm 1.9$ & $0.008^{*}$ \\
\hline$\%$ Fat & $37.9 \pm 2.0$ & $45.9 \pm 1.3$ & $0.002^{*}$ \\
\hline Total cholesterol (mmol I-1) & $5.0 \pm 0.1$ & $4.8 \pm 0.1$ & 0.34 \\
\hline LDL cholesterol (mmol $\left.{ }^{-1}\right)$ & $2.8 \pm 0.1$ & $2.6 \pm 0.1$ & 0.27 \\
\hline HDL cholesterol (mmol $\left.{ }^{-1}\right)$ & $1.6 \pm 0.1$ & $1.7 \pm 0.1$ & 0.53 \\
\hline Triglycerides $\left(\mathrm{mmol} \mathrm{I}^{-1}\right)$ & $1.1 \pm 0.1$ & $0.8 \pm 0.1$ & 0.17 \\
\hline Fasting glucose $\left(\mathrm{mmol} \mathrm{I}^{-1}\right)$ & $5.3 \pm 0.1$ & $5.3 \pm 0.1$ & 0.89 \\
\hline Serum creatinine $\left(\mu \mathrm{mol} \mathrm{I}^{-1}\right)$ & $82.2 \pm 2.6$ & $74.2 \pm 2.6$ & 0.06 \\
\hline eGFR $\left(\mathrm{ml} \mathrm{min}{ }^{-1}\right.$ per $\left.1.73 \mathrm{~m}^{2}\right)$ & $92.9 \pm 3.3$ & $97.4 \pm 4.0$ & 0.39 \\
\hline Baseline BA diameter (mm) & $3.5 \pm 0.1$ & $3.6 \pm 0.1$ & 0.82 \\
\hline FMD (\%) & $9.2 \pm 0.3$ & $5.4 \pm 0.2$ & $<0.001^{*}$ \\
\hline NMD (\%) & $17.5 \pm 1.1$ & $15.0 \pm 1.4$ & 0.18 \\
\hline FMD/NMD ratio & $0.55 \pm 0.03$ & $0.43 \pm 0.05$ & 0.06 \\
\hline Office SBP (mm Hg) & $122.3 \pm 2.5$ & $128.7 \pm 2.8$ & 0.10 \\
\hline Office DBP (mm Hg) & $77.8 \pm 1.6$ & $82.0 \pm 1.9$ & 0.11 \\
\hline Office SBPV STD (mm Hg) & $5.1 \pm 0.6$ & $6.5 \pm 0.8$ & 0.21 \\
\hline Office DBPV STD $(\mathrm{mm} \mathrm{Hg})$ & $3.7 \pm 0.6$ & $5.3 \pm 0.6$ & 0.07 \\
\hline Office SBPV CV (\%) & $4.2 \pm 0.5$ & $4.8 \pm 0.5$ & 0.44 \\
\hline Office DBPV CV (\%) & $4.7 \pm 0.8$ & $6.4 \pm 0.8$ & 0.13 \\
\hline 24-h SBP (mm Hg) & $127.8 \pm 2.3$ & $127.9 \pm 3.0$ & 0.97 \\
\hline 24-h DBP (mm Hg) & $77.7 \pm 1.5$ & $79.7 \pm 2.5$ & 0.51 \\
\hline 24-h SBPV (mm Hg) & $8.1 \pm 0.3$ & $9.4 \pm 0.5$ & $0.04^{*}$ \\
\hline 24-h DBPV (mm Hg) & $7.5 \pm 0.3$ & $8.4 \pm 0.5$ & 0.14 \\
\hline 24-h rate of SBPV $\left(\mathrm{mm} \mathrm{Hg} \mathrm{min}^{-1}\right)$ & $0.24 \pm 0.01$ & $0.27 \pm 0.01$ & 0.14 \\
\hline 24-h rate of DBPV $\left(\mathrm{mm} \mathrm{Hg} \mathrm{min}^{-1}\right)$ & $0.21 \pm 0.01$ & $0.24 \pm 0.01$ & 0.10 \\
\hline
\end{tabular}

Abbreviations: BA, brachial artery; BMI, body mass index; BP, blood pressure; CV, coefficient of variation; DBP, diastolic blood pressure; DBPV, diastolic blood pressure variability; eGFR, estimated glomerular filtration rate; FMD, flow-mediated vasodilation; HDL, high-density lipoprotein; LDL, low-density lipoprotein; NMD, nitroglycerin-mediated vasodilation; SBP, systolic blood pressure; SBPV, systolic blood pressure variability; STD, standard deviation. *denotes significant difference between endothelial function groups.

parameters when classified according to their \%FMD. Fat mass and $\%$ body fat were significantly higher in the decreased endothelial function group. BMI and the prevalence of females also tended to be higher in the decreased endothelial function group, but not significantly. A trend for lower serum creatinine levels in the decreased endothelial function group was also found. There were no significant differences between the normal and decreased endothelial function groups for mean BP levels obtained from office measures or ABPM. For measures of visit-to-visit office BPV, the s.d. of DBP across visits tended to be higher in the decreased endothelial function group, however, this difference did not reach statistical significance. For measures of BPV obtained during ABPM, 24-h SBP variability (SBPV) was significantly higher in the decreased endothelial function group.

Table 2 shows the comparisons between normal and decreased endothelial function groups for all clinical characteristics and BP 
Table 2 Clinical characteristics and BP parameters for endothelial function groups classified according to FMD/NMD ratio

\begin{tabular}{|c|c|c|c|}
\hline Variable & $\begin{array}{c}\text { Normal } \\
\text { endothelial } \\
\text { function } \\
\text { FMD/NMD } \\
\text { ratio } \geqslant 0.48 \\
(\mathrm{n}=18)\end{array}$ & $\begin{array}{c}\text { Decreased } \\
\text { endothelial } \\
\text { function } \\
\text { FMD/NMD } \\
\text { ratio <0.48 } \\
(\mathrm{n}=18)\end{array}$ & P-value \\
\hline Age (years) & $53.0 \pm 1.8$ & $50.6 \pm 1.0$ & 0.25 \\
\hline Male (\%)/female (\%) & $\begin{array}{l}7(39 \%) / \\
11(61 \%)\end{array}$ & $\begin{array}{l}2(11 \%) / \\
16(89 \%)\end{array}$ & 0.05 \\
\hline Antihypertensive medication (\%) & 33.3 & 33.3 & 1.00 \\
\hline $\mathrm{BMI}\left(\mathrm{kg} \mathrm{m}^{-2}\right)$ & $31.9 \pm 1.4$ & $33.2 \pm 1.2$ & 0.48 \\
\hline Fat-free mass (kg) & $54.7 \pm 2.6$ & $53.0 \pm 1.9$ & 0.59 \\
\hline Fat mass (kg) & $35.0 \pm 3.0$ & $42.4 \pm 2.6$ & 0.07 \\
\hline$\%$ Fat & $38.3 \pm 2.1$ & $44.9 \pm 1.5$ & $0.01^{*}$ \\
\hline Total cholesterol $\left(\mathrm{mmoll}^{-1}\right)$ & $5.0 \pm 0.1$ & $4.7 \pm 0.1$ & 0.11 \\
\hline LDL cholesterol $\left(\mathrm{mmol} \mathrm{I}^{-1}\right)$ & $2.9 \pm 0.1$ & $2.5 \pm 0.1$ & 0.08 \\
\hline HDL cholesterol $\left(\mathrm{mmol} \mathrm{I}^{-1}\right)$ & $1.6 \pm 0.1$ & $1.7 \pm 0.1$ & 0.69 \\
\hline Triglycerides $\left(\mathrm{mmol} \mathrm{I}^{-1}\right)$ & $1.0 \pm 0.1$ & $0.9 \pm 0.1$ & 0.35 \\
\hline Fasting glucose $\left(\mathrm{mmol} \mathrm{I}^{-1}\right)$ & $5.2 \pm 0.1$ & $5.3 \pm 0.1$ & 0.76 \\
\hline Serum creatinine $\left(\mu \mathrm{mol} \mathrm{I}{ }^{-1}\right)$ & $73.2 \pm 2.2$ & $62.5 \pm 1.5$ & $0.004^{*}$ \\
\hline eGFR $\left(\mathrm{ml} \mathrm{min}-1\right.$ per $\left.1.73 \mathrm{~m}^{2}\right)$ & $90.2 \pm 3.7$ & $99.4 \pm 3.3$ & 0.07 \\
\hline Baseline BA diameter (mm) & $3.6 \pm 0.1$ & $3.6 \pm 0.1$ & 0.99 \\
\hline FMD (\%) & $8.0 \pm 0.5$ & $6.8 \pm 0.4$ & 0.11 \\
\hline NMD (\%) & $12.6 \pm 1.0$ & $19.4 \pm 1.0$ & $<0.001 *$ \\
\hline FMD/NMD ratio & $0.66 \pm 0.04$ & $0.35 \pm 0.01$ & $<0.001^{*}$ \\
\hline Office SBP (mm Hg) & $122.7 \pm 2.1$ & $127.8 \pm 1.8$ & 0.19 \\
\hline Office DBP (mm Hg) & $78.1 \pm 1.0$ & $81.4 \pm 2.1$ & 0.17 \\
\hline Office SBPV STD (mm Hg) & $4.8 \pm 0.7$ & $6.6 \pm 0.7$ & 0.09 \\
\hline Office DBPV STD (mm Hg) & $3.4 \pm 0.8$ & $5.0 \pm 0.6$ & 0.22 \\
\hline Office SBPV CV (\%) & $3.9 \pm 0.6$ & $5.0 \pm 0.5$ & 0.15 \\
\hline Office DBPV CV (\%) & $5.0 \pm 0.9$ & $6.0 \pm 0.7$ & 0.41 \\
\hline 24-h SBP (mm Hg) & $125.8 \pm 2.2$ & $129.8 \pm 2.9$ & 0.29 \\
\hline 24-h DBP (mm Hg) & $79.6 \pm 1.3$ & $77.8 \pm 2.6$ & 0.54 \\
\hline 24-h SBPV (mm Hg) & $8.1 \pm 0.3$ & $9.3 \pm 0.4$ & 0.06 \\
\hline 24-h DBPV (mm Hg) & $7.4 \pm 0.3$ & $8.6 \pm 0.4$ & $0.04^{*}$ \\
\hline 24-h rate of SBPV $\left(\mathrm{mm} \mathrm{Hg} \mathrm{min}^{-1}\right)$ & $0.23 \pm 0.01$ & $0.27 \pm 0.01$ & $0.02^{*}$ \\
\hline 24-h rate of DBPV $\left(\mathrm{mm} \mathrm{Hg} \mathrm{min}^{-1}\right)$ & $0.20 \pm 0.01$ & $0.25 \pm 0.01$ & $0.01^{*}$ \\
\hline
\end{tabular}

Abbreviations: $\mathrm{BA}$, brachial artery; $\mathrm{BMI}$, body mass index; $\mathrm{BP}$, blood pressure; $\mathrm{CV}$, coefficient of variation; DBP, diastolic blood pressure; DBPV, diastolic blood pressure variability; eGFR, estimated glomerular filtration rate; FMD, flow-mediated vasodilation; HDL, high-density lipoprotein; LDL, low-density lipoprotein; NMD, nitroglycerin-mediated vasodilation; SBP, systolic blood pressure; SBPV, systolic blood pressure variability; STD, standard deviation. denotes significant difference between endothelial function groups.

parameters when classified according to their FMD/NMD ratio. Fat mass and $\%$ body fat were higher in the decreased endothelial function group, however, this difference was only significant for \% body fat. The decreased endothelial function group also showed nonsignificant trends for a higher prevalence of females, lower low-density lipoprotein cholesterol, lower serum creatinine levels and higher eGFR. For measures of visit-to-visit office BPV, the s.d. of SBP across visits tended to be higher in the decreased endothelial function group, however, this difference did not reach statistical significance. For measures of BPV obtained during ABPM, 24-h DBP variability (DBPV), the rate of 24-h SBPV and the rate of 24-h DBPV were all significantly higher in the decreased endothelial function group. Also, 24-h SBPV tended to be higher in the decreased endothelial function group, however, this difference was not statically significant.
When participants were divided into normal (\%NMD $\geqslant 16 \%$, $n=18$ ) or decreased (\%NMD $<16 \%, n=18$ ) smooth muscle-dependent dilation groups according to their \%NMD, there were no significant differences between the two groups for mean BP levels and all BPV indices.

The regression and Pearson correlation coefficients from univariate regression analyses of all clinical and BP variables significantly associated with either \%FMD, \%NMD or FMD/NMD ratio are provided in Table 3. Triglyceride levels and female gender were both significantly associated with \%FMD. Mean BP levels and BPV indices showed no associations with \%FMD. Total cholesterol, serum creatinine, eGFR, 24-h DBPV and the rate of 24-h DBPV were all significantly associated with \%NMD. The positive values of the Pearson correlation coefficients indicate that higher BPV was associated with higher \%NMD. The s.d. of SBP across office visits, the coefficient of variation of SBP across office visits, 24-h DBPV, the rate of 24-h SBPV and the rate of 24-h DBPV were all significantly associated with the FMD/NMD ratio. The negative values of the Pearson correlation coefficients indicate that higher BPV was associated with a lower FMD/NMD ratio. All remaining clinical variables and mean BP levels showed no significant associations with \%NMD or the FMD/NMD ratio.

The regression and partial correlation coefficients from the multivariate regression model are provided in Table 4. Serum creatinine, eGFR, 24-h DBPV and the rate of 24-h DBPV all remained significantly associated with \%NMD. For the FMD/NMD ratio, the s.d. of SBP across office visits, the coefficient of variation of SBP across office visits, 24-h DBPV, the rate of 24-h SBPV and the rate of 24-h DBPV all remained significantly associated with the FMD/NMD ratio after adjusting for age, BMI and mean BP levels.

\section{DISCUSSION}

In this study, we investigated the relationship between measures of endothelial-dependent and -independent vasodilation and BPV in a cohort of African Americans. To fully elucidate this relationship, two measures of BPV were used: visit-to-visit BPV, reflective of long-term BP fluctuations over days and weeks; and 24-h BPV, reflective of short-term BP fluctuations over hours. To the best of our knowledge, our study is the first to report on the relationship between measures of endothelial function and visit-to-visit BPV in any population. Moreover, although some studies have investigated the relationship between endothelial function and variables derived from $A B P M,{ }^{21,22}$ never has the relationship between endothelial function and fluctuations in BP from measurement to measurement during ABPM been investigated. Thus, our study is also the first to report on the relationship between measures of endothelial function and 24-h BPV in any population. Our results show that African Americans with decreased endothelial function, classified using either \%FMD or the FMD/NMD ratio, had higher 24-h BPV. Moreover, while not statistically significant, the decreased endothelial function group also had a trend for higher visitto-visit BPV. When analyzed as continuous variables, our results show that higher visit-to-visit SBPV, and 24-h SBPV and DBPV were all significantly associated with a lower FMD/NMD ratio, independent of age, BMI and mean BP. The use of the FMD/NMD ratio as a marker of endothelial function is predicated off the assumption that NMD is representative of the maximal achievable diameter of the BA; hence, the lower the FMD/NMD ratio the less that an individual attains of their maximal vasodilatory capacity. Therefore, our finding that high $\mathrm{BPV}$ is associated with a lower FMD/NMD ratio may indicate that endothelial-specific vasodilatory mechanisms are impaired in African Americans who exhibit high BPV. 
Table 3 Univariate regression analyses predicting vascular function measures

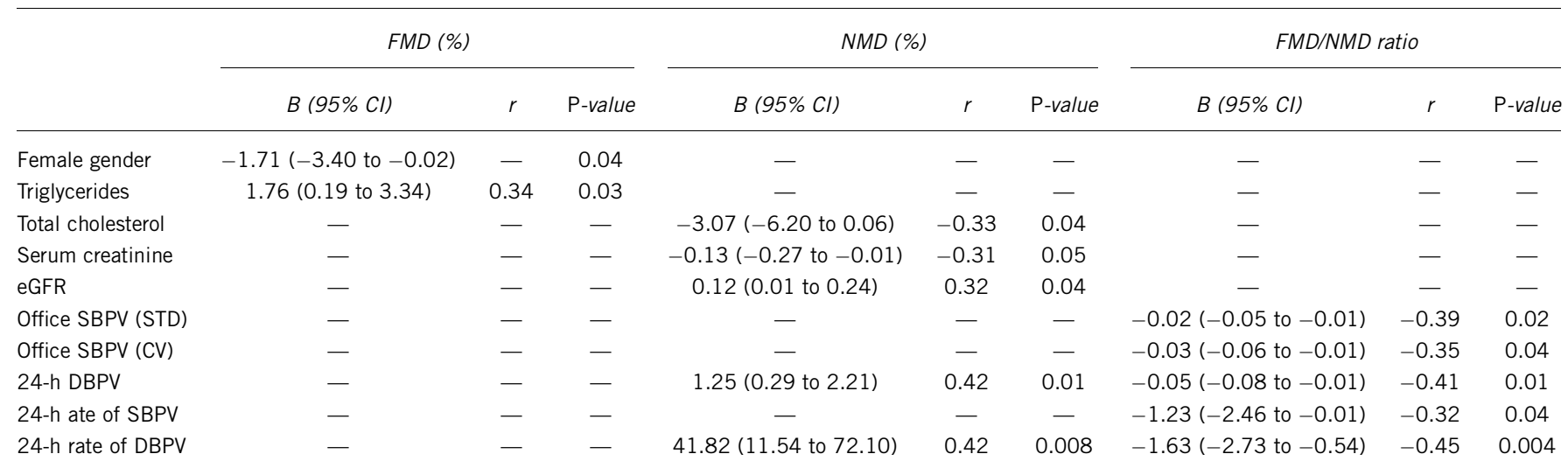

Abbreviations: B, unstandardized regression coefficient; Cl, confidence interval; CV, coefficient of variation; DBPV, diastolic blood pressure variability; eGFR, estimated glomerular filtration rate; FMD, flow-mediated vasodilation; NMD, nitroglycerin-mediated vasodilation; r, Pearson correlation coefficient; SBPV, systolic blood pressure variability.

Table 4 Multivariate regression analyses predicting vascular function measures

\begin{tabular}{|c|c|c|c|c|c|c|c|c|c|}
\hline & \multicolumn{3}{|c|}{$F M D(\%)$} & \multicolumn{3}{|c|}{$N M D(\%)$} & \multicolumn{3}{|c|}{ FMD/NMD ratio } \\
\hline & $B(95 \% C l)$ & $\begin{array}{c}\text { Partial } \\
\text { correlation }\end{array}$ & P-value & $B(95 \% C l)$ & $\begin{array}{c}\text { Partial } \\
\text { correlation }\end{array}$ & P-value & $B(95 \% C l)$ & $\begin{array}{c}\text { Partial } \\
\text { correlation }\end{array}$ & $\mathrm{P}$-value \\
\hline Female gender & $-1.51(-3.48$ to 0.46$)$ & - & 0.12 & - & - & - & - & - & - \\
\hline Triglycerides & $1.47(-0.33$ to 3.28$)$ & 0.29 & 0.10 & - & - & - & - & - & - \\
\hline Total cholesterol & - & - & - & $-2.42(-6.21$ to 1.35$)$ & -0.26 & 0.11 & - & - & - \\
\hline Serum creatinine & - & - & - & $-0.14(-0.28$ to -0.01$)$ & -0.35 & 0.03 & - & - & - \\
\hline eGFR & - & - & - & $0.15(0.03$ to 0.28$)$ & 0.43 & 0.02 & - & - & - \\
\hline Office SBPV (STD) & - & - & - & - & - & - & $-0.03(-0.05$ to -0.01$)$ & -0.42 & 0.03 \\
\hline Office SBPV (CV) & - & - & - & - & - & - & $-0.04(-0.06$ to -0.01$)$ & -0.39 & 0.03 \\
\hline 24-h DBPV & - & - & - & $1.33(0.33$ to 2.32$)$ & 0.44 & 0.01 & $-0.04(-0.08$ to -0.01$)$ & -0.41 & 0.02 \\
\hline 24-h rate of SBPV & - & - & - & - & - & - & $-1.46(-2.88$ to -0.03$)$ & -0.30 & 0.04 \\
\hline 24-h rate of DBPV & - & - & - & $40.90(8.52$ to 73.21$)$ & 0.42 & 0.01 & $-1.59(-2.81$ to -0.37$)$ & -0.44 & 0.01 \\
\hline
\end{tabular}

Abbreviations: B, unstandardized regression coefficient; BMI, body mass index; Cl, confidence interval; CV, coefficient of variation; DBP, diastolic blood pressure; DBPV, diastolic blood pressure variability; eGFR, estimated glomerular filtration rate; FMD, flow-mediated vasodilation; NMD, nitroglycerin-mediated vasodilation; SBP, systolic blood pressure; SBPV, systolic blood pressure variability.

The listed variables were entered separately into a multivariate regression model containing age, BMI, SBP and DBP.

As a result of the cross-sectional design of this study, it is difficult to ascertain whether high BPV precedes impaired endothelial function or vice versa. The most compelling evidence for high BPV being a cause rather than a consequence of impaired endothelial function stems from animal studies using sinoaortic-denervation (SAD) rats. SAD is a procedure whereby the arterial baroreflex system is interrupted, causing significant increases in BPV without eliciting changes in mean BP levels. SAD rats, therefore, are considered to be an animal model for BPV, as the physiological changes caused by high BPV can be investigated without the confounding effect of hypertension. ${ }^{23}$ Previous studies in SAD rats have shown that impaired endothelial function is present in this animal model of high BPV after SAD, thus providing experimental evidence in support of the hypothesis that high BPV may be a contributing factor in the pathogenesis impaired endothelial function. ${ }^{24,25}$ It has also been reported that inflammatoryrelated factors are increased in SAD rats, and that long-term antiinflammatory and antioxidant treatment prevents SAD-induced organ damage; suggestive that inflammation could be the underlying mechanism facilitating endothelial dysfunction in SAD rats. ${ }^{26}$ In this study, our finding of increased BPV in African Americans categorized as having decreased endothelial function provides the first clinical data in human subjects that high BPV may be associated with impaired endothelial function. We have also previously reported in this cohort of African Americans that high BPV is associated with increased circulating levels of biomarkers for systemic inflammation. ${ }^{9}$ Both findings coincide with experimental findings in SAD rats, and could strengthen the hypothesis that BPV confers a greater CV risk through the induction of vascular damage, which in turn may attenuate endothelial function. Of course, prospective longitudinal studies are needed to confirm this hypothesis.

It has been proposed that the FMD/NMD ratio is the best available marker of endothelial function because differences in the VSM response to $\mathrm{NO}$ can be accounted for. ${ }^{27,28}$ The FMD/NMD ratio was first utilized in 1993, when Celermajer et al. ${ }^{29}$ showed that the FMD/NMD ratio was decreased in adult smokers when compared with adult non-smokers. Subsequent studies utilizing the FMD/NMD ratio have reported that it is decreased in essential hypertension and diabetes, and is associated with the number of CV risk factors. ${ }^{27,30,31}$ The most supportive data for the FMD/NMD ratio serving as the best available marker of endothelial function, comes from a study by Chan et $a .^{32}$ who showed that the FMD/NMD ratio was a more powerful predictor of vascular events than FMD. This finding led investigators 
to suggest that the portion of vascular dilation related solely to endothelial mechanisms and not smooth muscle mechanisms is most important, and that the calculation of the FMD/NMD ratio is advantageous for determining the true prognostic value of endothelial function independent of smooth muscle function. In this study, when analyzed as a continuous variable, the FMD/NMD ratio was negatively associated with measures of visit-to-visit and 24-h BPV. If the FMD/ NMD ratio is indeed the best available marker of endothelial function; our study findings could provide important information regarding the clinical importance of monitoring BPV. Unfortunately, although the $\mathrm{FMD} / \mathrm{NMD}$ ratio is used by some investigators, it is frequently not reported in the literature despite sound rationale and previous data supporting its use, thus its true prognostic value has not be clearly established. Therefore, although provocative, our findings may be tempered to some extent by a lack of studies investigating the clinical and prognostic importance of the FMD/NMD ratio.

In this study, although differences in BPV were observed between endothelial function groups classified according to \%FMD, when $\%$ FMD was analyzed as a continuous variable we found no associations between \%FMD and any measure of BPV. This finding is contrary to the significant associations found between the FMD/NMD ratio and measures of both visit-to-visit and 24-h BPV in our study population. Reasons for significant associations only being found for the FMD/ NMD ratio and not \%FMD are unclear, but could, in part, be confounded by differences in the VSM response to NO. We found that high 24-h DBPV was associated with an increased VSM response to nitroglycerin. Considering the lack of association between FMD and $\mathrm{BPV}$, the negative association between the FMD/NMD ratio and BPV could be driven by the effect of BPV on VSM responsiveness to nitroglycerin rather than its deleterious effect on FMD. Consistent with this hypothesis, previous animal studies have reported differences in vascular reactivity to contractile and vasodilatory agonists between SAD rats and sham-operated rats. ${ }^{33}$ Interestingly, Rocha et al..$^{33}$ found that expression of integral membrane proteins responsible for the formation of gap junctions in VSM were increased in SAD rats; leading investigators to propose that the differences in vascular reactivity observed between $\mathrm{SAD}$ rats and sham-operated rats could be the result of increased gap junction communication in the SAD rats. Given that NO-induced vasodilation has previously been shown to be dependent on cell-to-cell diffusion of cyclic guanosine monophosphate (cGMP) through gap junctions, ${ }^{34}$ we hypothesize that a potential mechanism for the association between high BPV and a greater vasodilatory response to nitroglycerin could be the result of increased gap junction communication in the arteries of individual's with high BPV. However, although we think this hypothesis is reasonable, it should be acknowledged that this study cannot confirm the real mechanism contributing to the observed association between NMD and BPV. Furthermore, more clinical studies will be needed to further elucidate the relationship between BPV and vascular reactivity to vasodilatory agonists, as well as contractile agonists.

Several limitations of this study must be noted when interpreting our findings. First, our sample size is small. However, because of our extensive exclusion criteria, many confounding variables that may influence vascular function were controlled for. Second, only African Americans were included in the study. Therefore, are study findings are not generalizable to other race-ethnicities. Third, the reproducibility of BPV may be poor because of the large influence of daily activity on BPV. However, recent reports have shown that both visit-to-visit and 24-h BPV have fairly good reproducibility. ${ }^{35,36}$ Finally, our crosssectional study design allowed us to assess the relationship between vascular function and BPV, but not to assess cause-effect relationships.
In conclusion, our study provides novel information by assessing the relationship between BPV and measures of vascular function for the first time in humans. Our findings provide some evidence that BPV may be in increased in African Americans with decreased endothelial function and are associated with the VSM response to NO. Additional research is needed to confirm these results and determine the clinical relevance of the FMD/NMD ratio.

\section{CONFLICT OF INTEREST}

The authors declare no conflict of interest.

\section{ACKNOWLEDGEMENTS}

This research was supported by NIH/NHLBI Grant RO1 HL085497 (PI, Michael D Brown) and by NIH/NIA Grant KO1 AG019640 (PI, Michael D Brown).

1 Kikuya M, Ohkubo T, Asayama K, Metoki H, Obara T, Saito S, Hashimoto J, Totsune $\mathrm{K}$, Hoshi H, Satoh H, Imai Y. Ambulatory blood pressure and 10-year risk of cardiovascular and noncardiovascular mortality: the Ohasama study. Hypertension 2005; 45: 240-245.

2 Mancia G, Bombelli M, Facchetti R, Madotto F, Corrao G, Trevano FQ, Grassi G, Sega R. Long-term prognostic value of blood pressure variability in the general population: results of the Pressioni Arteriose Monitorate e Loro Associazioni Study. Hypertension 2007; 49: 1265-1270.

3 Muntner P, Shimbo D, Tonelli M, Reynolds K, Arnett DK, Oparil S. The relationship between visit-to-visit variability in systolic blood pressure and all-cause mortality in the general population: findings from NHANES III, 1988 to 1994. Hypertension 2011; 57: 160-166.

4 Rothwell PM, Howard SC, Dolan E, O'Brien E, Dobson JE, Dahlof B, Sever PS, Poulter NR. Prognostic significance of visit-to-visit variability, maximum systolic blood pressure, and episodic hypertension. Lancet 2010; 375: 895-905.

5 Kim KI, Lee JH, Chang HJ, Cho YS, Youn TJ, Chung WY, Chae IH, Choi DJ, Park KU, $\mathrm{Kim} \mathrm{CH}$. Association between blood pressure variability and inflammatory marker in hypertensive patients. Circ J 2008; 72: 293-298.

6 Abramson JL, Lewis C, Murrah NV, Anderson GT, Vaccarino V. Relation of C-reactive protein and tumor necrosis factor-alpha to ambulatory blood pressure variability in healthy adults. Am J Cardiol 2006; 98: 649-652.

7 Tatasciore A, Zimarino $M$, Renda $G$, Zurro $M$, Soccio $M$, Prontera C, Emdin $M$, Flacco M, Schillaci G, DE CR. Awake blood pressure variability, inflammatory markers and target organ damage in newly diagnosed hypertension. Hypertens Res 2008; 31: 2137-2146.

8 Veerabhadrappa P, Diaz KM, Feairheller DL, Sturgeon KM, Williamson S, Crabbe DL, Kashem A, Ahrensfield D, Brown MD. Enhanced blood pressure variability in a high cardiovascular risk group of African Americans: FIT4 Life Study. J Am Soc Hypertens 2010; 4: 187-195.

9 Green DJ, Jones H, Thijssen D, Cable NT, Atkinson G. Flow-mediated dilation and cardiovascular event prediction: does nitric oxide matter? Hypertension 2011; 57: 363-369.

10 Lind L, Berglund L, Larsson A, Sundstrom J. Endothelial function in resistance and conduit arteries and 5-year risk of cardiovascular disease. Circulation 2011; 123: 1545-1551.

11 Yeboah J, Folsom AR, Burke GL, Johnson C, Polak JF, Post W, Lima JA, Crouse JR, Herrington DM. Predictive value of brachial flow-mediated dilation for incident cardiovascular events in a population-based study: the multi-ethnic study of atherosclerosis. Circulation 2009; 120: 502-509.

12 Levey AS, Coresh J, Greene T, Stevens LA, Zhang YL, Hendriksen S, Kusek JW, Van LF. Using standardized serum creatinine values in the modification of diet in renal disease study equation for estimating glomerular filtration rate. Ann Intern Med 2006; 145: 247-254.

13 Krauss RM, Deckelbaum RJ, Ernst N, Fisher E, Howard BV, Knopp RH, Kotchen T, Lichtenstein AH, McGill HC, Pearson TA, Prewitt TE, Stone NJ, Horn LV, Weinberg R. Dietary guidelines for healthy American adults. A statement for health professionals from the Nutrition Committee, American Heart Association. Circulation 1996; 94: 1795-1800.

14 Chobanian AV, Bakris GL, Black HR, Cushman WC, Green LA, Izzo Jr JL, Jones DW, Materson BJ, Oparil S, Wright Jr JT, Roccella EJ. Seventh report of the Joint National Committee on Prevention, Detection, Evaluation, and Treatment of High Blood Pressure. Hypertension 2003; 42: 1206-1252.

15 Diaz KM, Feairheller DL, Sturgeon KM, Veerabhadrappa P, Williamson ST, Crabbe DL, Brown MD. Increased nitric oxide and attenuated diastolic blood pressure variability in African Americans with mildly impaired renal function. Int J Hypertens 2010; 2010: 137206.

16 Mena L, Pintos S, Queipo NV, Aizpurua JA, Maestre G, Sulbaran T. A reliable index for the prognostic significance of blood pressure variability. J Hypertens 2005; 23: 505-511. 
17 Pierdomenico SD, Di NM, Esposito AL, Di MR, Ballone E, Lapenna D, Cuccurullo F. Prognostic value of different indices of blood pressure variability in hypertensive patients. Am J Hypertens 2009; 22: 842-847.

18 Zakopoulos NA, Tsivgoulis G, Barlas G, Papamichael C, Spengos K, Manios E, Ikonomidis I, Kotsis V, Spiliopoulou I, Vemmos K, Mavrikakis M, Moulopoulos SD. Time rate of blood pressure variation is associated with increased common carotid artery intima-media thickness. Hypertension 2005; 45: 505-512.

19 Veerabhadrappa P, Diaz KM, Kashem MA, Crabbe DL, Feairheller DL, Sturgeon KM, Williamson S, Brown MD. Endothelial dysfunction in prehypertensive African Americans with masked hypertension. Am J Hypertens 2011 (doi:10.1038/ajh.2011.103).

20 Corretti MC, Anderson TJ, Benjamin EJ, Celermajer D, Charbonneau F, Creager MA, Deanfield J, Drexler H, Gerhard-Herman M, Herrington D, Vallance P, Vita J, Vogel R. Guidelines for the ultrasound assessment of endothelial-dependent flow-mediated vasodilation of the brachial artery: a report of the International Brachial Artery Reactivity Task Force. J Am Coll Cardiol 2002; 39: 257-265.

21 Gupta AK, Cornelissen G, Greenway FL, Dhoopati V, Halberg F, Johnson WD. Abnormalities in circadian blood pressure variability and endothelial function: pragmatic markers for adverse cardiometabolic profiles in asymptomatic obese adults. Cardiovasc Diabetol 2010; 9: 58.

22 Higashi Y, Nakagawa K, Kimura M, Noma K, Hara K, Sasaki S, Goto C, Oshima T, Chayama K, Yoshizumi M. Circadian variation of blood pressure and endothelial function in patients with essential hypertension: a comparison of dippers and nondippers. J Am Coll Cardiol 2002; 40: 2039-2043.

23 Norman Jr RA, Coleman TG, Dent AC. Continuous monitoring of arterial pressure indicates sinoaortic denervated rats are not hypertensive. Hypertension 1981; 3: 119-125.

24 Eto M, Toba K, Akishita M, Kozaki K, Watanabe T, Kim S, Hashimoto M, Sudoh N, Yoshizumi M, Ouchi Y. Reduced endothelial vasomotor function and enhanced neointimal formation after vascular injury in a rat model of blood pressure liability. Hypertens Res 2003; 26: 991-998.

25 Miao CY, Tao X, Gong K, Zhang SH, Chu ZX, Su DF. Arterial remodeling in chronic sinoaortic-denervated rats. J Cardiovasc Pharmacol 2001; 37: 6-15.

26 Zhang C, Chen H, Xie HH, Shu H, Yuan WJ, Su DF. Inflammation is involved in the organ damage induced by sinoaortic denervation in rats. J Hypertens 2003; 21: 2141-2148.
27 Oshima T, Ozono R, Yano Y, Higashi Y, Teragawa H, Miho N, Ishida T, Ishida M, Yoshizumi M, Kambe M. Beneficial effect of T-type calcium channel blockers on endothelial function in patients with essential hypertension. Hypertens Res 2005; 28 : 889-894.

28 Wiltshire EJ, Gent R, Hirte C, Pena A, Thomas DW, Couper JJ. Endothelial dysfunction relates to folate status in children and adolescents with type 1 diabetes. Diabetes 2002; 51: 2282-2286.

29 Celermajer DS, Sorensen KE, Georgakopoulos D, Bull C, Thomas O, Robinson J, Deanfield JE. Cigarette smoking is associated with dose-related and potentially reversible impairment of endothelium-dependent dilation in healthy young adults. Circulation 199; 88: 2149-2155.

30 Heiss C, Lauer T, Dejam A, Kleinbongard P, Hamada S, Rassaf T, Matern S, Feelisch M, Kelm M. Plasma nitroso compounds are decreased in patients with endothelial dysfunction. J Am Coll Cardiol 2006; 47: 573-579.

31 Sibal L, Aldibbiat A, Agarwal SC, Mitchell G, Oates C, Razvi S, Weaver JU, Shaw JA, Home PD. Circulating endothelial progenitor cells, endothelial function, carotid intimamedia thickness and circulating markers of endothelial dysfunction in people with type 1 diabetes without macrovascular disease or microalbuminuria. Diabetologia 2009; 52: 1464-1473

32 Chan SY, Mancini GB, Kuramoto L, Schulzer M, Frohlich J, Ignaszewski A. The prognostic importance of endothelial dysfunction and carotid atheroma burden in patients with coronary artery disease. J Am Coll Cardiol 2003; 42: 1037-1043.

33 Rocha ML, Kihara AH, Davel AP, Britto LR, Rossoni LV, Bendhack LM. Blood pressure variability increases connexin expression in the vascular smooth muscle of rats. Cardiovasc Res 2008; 80: 123-130.

34 Christ GJ, Brink PR, Ramanan SV. Dynamic gap junctional communication: a delimiting model for tissue responses. Biophys J 1994; 67: 1335-1344.

35 Eguchi K, Hoshide S, Hoshide Y, Ishikawa S, Shimada K, Kario K. Reproducibility of ambulatory blood pressure in treated and untreated hypertensive patients. $J$ Hypertens 2010; 28: 918-924.

36 Howard SC, Rothwell PM. Reproducibility of measures of visit-to-visit variability in blood pressure after transient ischaemic attack or minor stroke. Cerebrovasc Dis 2009; 28: $331-340$. 